\title{
PALLIATIVE OPTIONS IN THE TREATMENT OF PANCREATIC ADENOCARCINOMA
}

\author{
Nikola Kolev, Valentin Ignatov, Anton Tonev, Elitsa Encheva, Shteryo Shterev, \\ Aleksandar Zlatarov, Dilyan Petrov, Krasimir Ivanov \\ First Clinic of Surgery, St. Marina University Hospital of Varna, \\ Medical University of Varna
}

\begin{abstract}
INTRODUCTION/BACKGROUND: The pancreatic adenocarcinoma (PA) is one of the most lethal malignancies being the fourth leading cause of cancer deaths worldwide. Despite the significant progress in diagnostic modalities the PA is still diagnosed at late stages when curative treatment is no longer possible. This unfavorable situation directs the clinician's attention towards palliation and quality of life improvement.

MATERIALS AND METHODS: We performed a literature review of the recent developments in the area of palliative care for pancreatic cancer patients regarding the management of obstructive jaundice, pain and intestinal obstruction. The experience of our clinic with conventional surgery and nonsurgical methods is analysed and presented.

OUR RESULTS: We report our experience with a group of 45 patients. One part of them received conventional surgical treatment, and the other one underwent endoscopic treatment. Both endoscopic stent insertion and surgical bypass appear to be effective treatments, but nonoperative palliation seems to be associated with lower complication rates, lower procedure-related mortality rates, and shorter initial periods of hospitalisation compared with surgical palliation. In one patient we performed recanalisation of a selfexpandable metallic stent by inserting a plastic stent into it. The jaundice was relieved successfully.

CONCLUSION: The management of PA patient remains a challenge in spite of the clear progress made in diagnostic studies, surgical and palliation treatments. Multidisciplinary approach is necessary to optimise the overall care. Considering the late diagnosis of the majority of PA patients, palliative techniques should not be neglected, but well mastered.
\end{abstract}

Keywords: pancreatic adenocarcinoma, palliative, surgery, endoscopic

\author{
Address for corespondence \\ Georgi Ivanov \\ First Clinic of Surgery, University Hospital "St. Marina" \\ Medical University of Varna \\ 1 Hr. Smirnenski Str. \\ 9000, Varna, Bulgaria \\ E-mail: ghivanov@abv.bg
}

Received: October 10, 2013

Accepted: December 18, 2013

\section{INTRODUCTION}

Pancreatic adenocarcinoma (PA) is one of the most fatal malignancies. Palliative care represents an important aspect of management in patients with pancreatic malignancy. In the last decades a huge progress was made in the diagnosis and palliative care of the advanced PA. Pancreatic adonocarcinoma is relatively common as it is the fourth leading cause of cancer-related mortality. The overall 5-year survival rate is $5 \%$, making this disease a model tumour in which to address the specialised care issues 
Nikola Kolev, Valentin Ignatov, Anton Tonev et al.

of palliative medicine. A multidisciplinary approach to this disease is therefore recommended.

\section{MATERIALS AND METHODS}

A review of the available literature data was performed. The endoscopic and surgical palliative procedures were analysed. We also report the clinic's experience with 45 patients for a period of 6 years (2006-2011). On the next table we present the most common symptoms of advanced pancreatic cancer.

\begin{tabular}{lc}
$\begin{array}{l}\text { Table } 1 \text { Presenting symptoms of advanced pancreatic } \\
\text { cancer (7) }\end{array}$ & \\
Symptom & Percentage \\
Abdominal Pain & $78-82$ \\
Anorexia & 64 \\
Early Satiety & 62 \\
Jaundice & $56-80$ \\
Sleep disorders & 54 \\
Weight loss & $66-84$ \\
Diabetes & 97 \\
Back Pain & 48 \\
Nausea and weight loss & $50-86$ \\
\hline
\end{tabular}

The most common symptoms are the jaundice, abdominal pain, weight loss and diabetes.

BILIARY OBSTRUCTION - Cholestasis - usually develops in patients with a tumour in the head of pancreas. The obstructive jaundice could be managed by endoscopic therapy as the preferred mode of palliation. In the past, staging laparotomy and biliary bypass were frequentlyperformed for unresectable PC of the head. During the last decades, the development of interventional radiology and endoscopy has allowed palliation of obstructive jaundice by the insertion of percutaneous or endoluminal stents with minimal morbidity and mortality. Jaundice can cause hepatic and renal failure and can also lead to dysfunction of the coagulation cascade. Therefore treatment of obstructive jaundice is one of the major aims of palliative therapy for carcinomas of the pancreatic head. Today, the most common treatments are endoscopic biliary stenting and surgical biliary bypass surgery with or without concomitant gastrojejunostomy. Biliary stenting can be done by ERCP (endoscopic retrograde cholangiopancreatography) or PTC (percutaneoustranshepatic Cholangiopa ncreatography). ERCP is an imaging technique used to diagnose diseases of hepatic and pancreaticobiliary origin. Special instruments are used to place stents. The major advantage of biliary stents is that the procedure used to place them is minimally invasive and well-tolerated by patients.

PAIN - About $70 \%$ of patients with unresectable PC develop clinically important pain during their lives. Pain is the main cause of the significant drop in quality and quantity of the life of these patients and good palliation is necessary as pain incidence and severity increases with disease progression. For the majority of patients, pain from PC can be managed with opioid analgesics.

Abdominal and back pain are more common in patients with distal PC and in patients with tumors infiltrating peri-pancreatic nerve tissue. For the majority of PC patients, pain is still controlled pharmacologically even if other modalities such as:

- surgical thoracoscopic splanchnicectomy,

- epidural anesthesia,

- subcutaneous injection with octreotide,

- hypofractionated-accelerated radiotherapy

- more recently photodynamic therapy

OUR RESULTS - Over a period of 6 years, we retrospectively analysed the clinical records of a total of 45 patients ( 19 women, 28 men) with unresectable, histologically proven adenocarcinoma of the pancreatic head or distal biliary tree who presented for palliative therapy in our department. The median age of the patients included was 63 years (range: $36-$ 89 years). At the time of diagnosis, obstructive jaundice was evident in 36 cases (76\%) and these patients therefore required palliative treatment. Patients with jaundice were divided into three groups based on the treatment they received:

Group 1: endoscopic stent placement without subsequent hepaticojejunostomy;

Group 2: preoperative stenting with subsequent palliative hepaticojejunostomy;

Group 3: hepaticojejunostomy without preoperative stenting. 
We determined the frequency of re-hospitalization for stent failure, stent-associated complications, and/or recurrent jaundice among the patients in Group 1. In 7 patients with obstructive jaundice, endoscopic stent implantation and/or surgery were impossible due to poor patient condition or changes in gastro-intestinal anatomy. In these 7 (19.5\%) patients, the obstructive jaundice was treated radiologically by percutaneous transhepatic biliary drainage (PTC). Morbidity showed significant differences among the three groups: patients who only underwent stenting (Group 1: 3/45, 6.38\%) had a significantly lower morbidity rate than patients treated with preoperative stenting and subsequent palliative hepaticojejunostomy (Group 2: 8/45, 17\%) while the comparison between patients who underwent hepaticojejunostomy without preoperative stenting did not reach the significant level (Group 3: 6/45, 10.6\%). In Group 1 , three patients developed cholangitis or pneumonia and had to be treated with antibiotics. Four patients had to have their stent changed during the initial hospital stay due to stent obstruction. The estimated mean interval between initial stent placement and stent replacement was $69.8 \pm 30.0$ days. In one patient we performed recanalisation of a self-expandable metallic stent by inserting a plastic stent into it. The jaundice was relieved successfully. Only two patients in Group 2 showed evidence of an anastomotic leak after hepaticojejunostomy and preoperative biliary stenting; of these two patients, one patient underwent reoperation and the other one was treated with ultrasound-guided drainage. The three cases of postoperative wound infection which occurred in Group 2 were all considered to be mild complications. In four patients who did not undergo preoperative biliary stenting (Group 3), leakage occurred at the anastomotic site after the hepaticojejunostomy. One of them underwent reoperation due to peritonitis and drainage failure. One patient in this group developed a pancreatic fistula after fine needle aspiration of the pancreatic tumor and one patient experienced postoperative wound infection. None of the patients who underwent surgery (Groups 2 and Group 3) had to be re-hospitalised for recurrent jaundice. Additionally, gastroenterostomy $(n=6 ; 12.8 \%)$ was only performed primarily in patients with evidence of duodenal obstruction without any increase in morbidity. In 3 pa- tients only duodenal obstruction after hepaticojejunostomy was observed and led to re-operation (gastroenterostomy). There were no significant differences in the mortality rates observed among the three patient groups (Group 1: 2.2\%; Group 2: 0\%; Group $3: 2.4 \% ; \mathrm{P}=0.459)$.

One death was attributed to cholangitis and subsequent sepsis and two other patients died due to reasons unrelated to the procedure carried out for their underlying disease.One patient in Group 3 died after hepaticojejunostomy due to leakage at the anastomotic site and subsequent sepsis (2.4\%).

OVERALL SURVIVAL - Overall survival was significantly different among the 3 groups of patients. In particular, median overall survival was significantly greater in patients who underwent pallia-
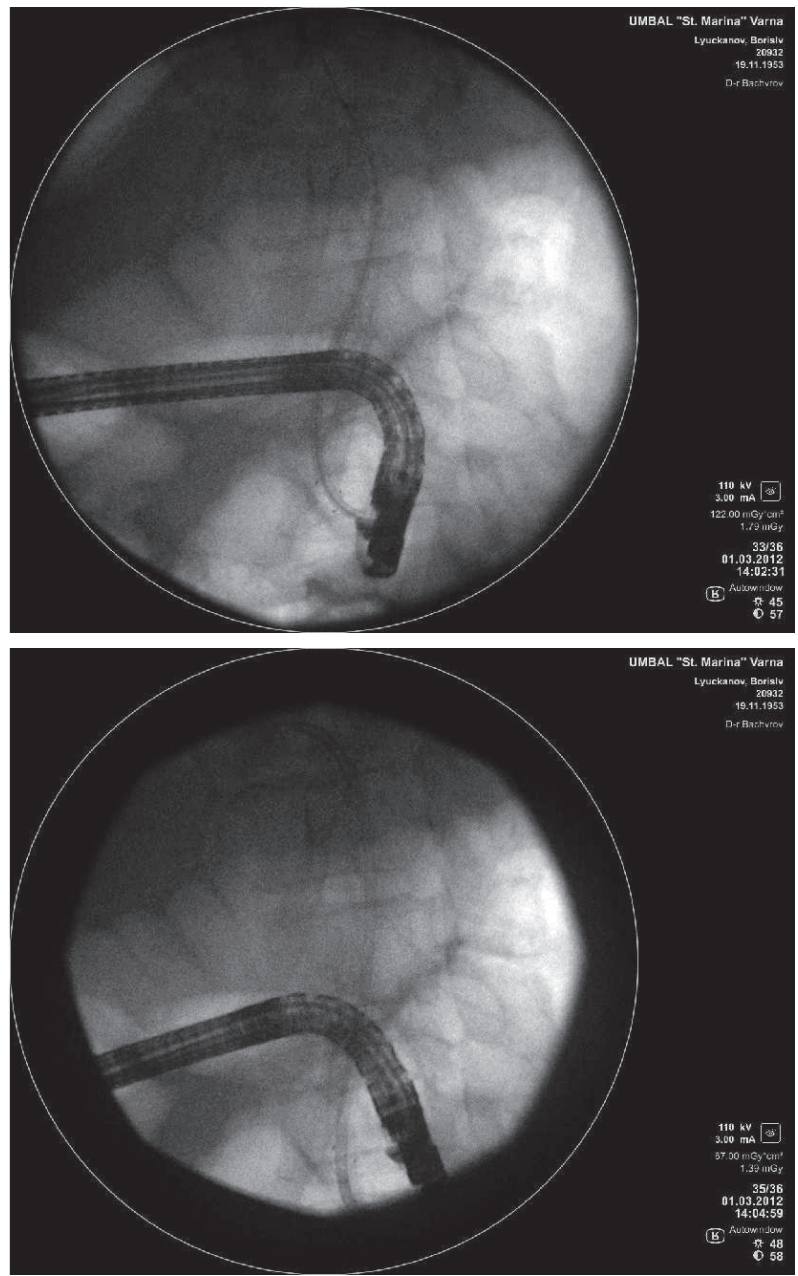


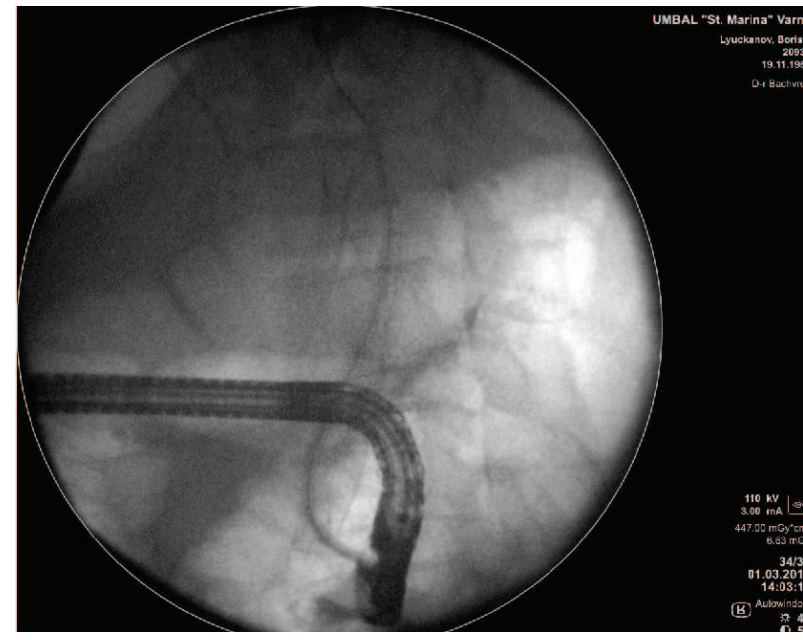

tive surgery with or without stent placement (Groups 2 and 3: 9.0 months) than in the patients who only underwent stent placement (Group 1: 5.1 months). When the outcomes of patients in Group 1 (stent) were compared to those of patients in Group 2 (stent and hepaticojejunostomy), we found that the patients in Group 2 had significantly longer median survival (9.4 months)

\section{CONCLUSION}

Pancreatic adenocarcinoma is a model illness that mandates the need for good supportive and palliative treatment. Pain may be linked with depression and anxiety. Interventional pain management technigues should not to be considered as last resort in pain management. Biliary obstruction can be successfully palliated with endoscopic stent placement in selected patients. Multidisciplinary approach to this disease is therefore recommended. Due to the low survival rate and late diagnosis this disease is a model tumour in which to address the specialised care issues of palliative medicine.

\section{REFERENCES}

1. Huibregtse K, Tytgat GN. Palliative treatment of obstructive jaundice by transpapillary introduction of large bore bile duct endoprosthesis. Gut 1982; 23:371-5

2. Shepherd HA, Royle G, Ross AP, Diba A, Arthur M, Colin- Jones D. Endoscopic biliary endoprosthesis in the palliation of malignant obstruction of the distal common bile duct: a randomized trial. Br J Surg 1988; 75:1166-8.

3. Lillemoe KD, Cameron JL, Hardacre JM, Sohn TA, Sauter PK, Coleman J, et al. Is prophylactic gastrojejunostomy indicated for unresectable periampullary cancer? A prospective randomized trial. Ann Surg 1999; 230:322-8.

4. Lillemoe KD, Pitt HA. Palliation. Surgical and otherwise. Cancer 1996; 78(Suppl 3):605-14.

5. Andersen JR, Sørensen SM, Kruse A, Rokkjaer M, Matzen P. Randomised trial of endoscopic endoprosthesis versus operative bypass in malignant obstructive jaundice. Gut 1989; 30:1132-5.

6. Bornman PC, Harries-Jones EP, Tobias R, Van Stiegmann G, Terblanche J. Prospective controlled trial of transhepatic biliary endoprosthesis versus bypass surgery for incurable carcinoma of head of pancreas. Lancet 1986; 327:69-71.

7. Valerie Lee, Haiying Cheng, Guangyuan Li, Muhammad Wasif Saif - Quality of life in patients with pancreatic cancer. JOP. J Pancreas (Online) 2012 mar 10. 